\title{
On Fairness Optimization for NOMA-Enabled Multi-Beam Satellite Systems
}

\author{
Anyue Wang ${ }^{1}$, Lei Lei ${ }^{1}$, Eva Lagunas ${ }^{1}$, Ana I. Pérez Neira ${ }^{2}$, Symeon Chatzinotas ${ }^{1}$, and Björn Ottersten ${ }^{1}$ \\ ${ }^{1}$ Interdisciplinary Centre for Security, Reliability and Trust (SnT), University of Luxembourg, Luxembourg \\ ${ }^{2}$ Center Tecnològic de Telecomunicacions de Catalunya (CTTC), Spain \\ Emails: \{anyue.wang; lei.lei; eva.lagunas; symeon.chatzinotas; bjorn.ottersten@uni.lu, ana.perez@cttc.es\}
}

\begin{abstract}
In a multi-beam satellite communication system, traffic requests are typically asymmetric across beams and highly heterogeneous among terminals. In practical operations, it is important to achieve a good match between the offered and requested traffic, i.e., to improve the performance of Offered Capacity to requested Traffic Ratio (OCTR). Due to satellites' payload constraints and limited flexibilities, it is a challenging task for resource optimization. In this paper, we tackle this issue by formulating a maxmin resource allocation problem, taking fairness into account such that the lowest OCTR can be maximized. To exploit the potential synergies, we introduce Non-Orthogonal Multiple Access (NOMA) to enable aggressive frequency reuse and mitigate intra-beam interference. Although NOMA has proven its capabilities in improving throughput and fairness in $5 \mathrm{G}$ terrestrial networks, for multi-beam satellite systems it is unclear if NOMA can help to enhance the OCTR performance, and hence is worth quantifying how much gain it can bring. To solve the problem, we design a suboptimal algorithm to firstly decompose the original problem into multiple convex subproblems by fixing power allocation for each beam, and secondly adjust beam power to improve the minimum OCTR in iterations. Numerical results show the convergence of the proposed algorithm and the superiority of the proposed NOMA scheme in max-min OCTR.
\end{abstract}

\section{INTRODUCTION}

From Cisco's prediction, the amount of wireless and mobile traffic will occupy $71 \%$ of total traffic by 2022 , and the traffic distribution will be heavily imbalanced over a wide range of geographical areas [1]. The upward trend and traffic asymmetry necessitate the development of new types of data-service techniques. With the advantages of wide coverage, service continuity, and fiber-like data transmission, using satellites to serve terrestrial terminals, e.g., satellite-based backhauling [2], is envisioned as one of the most promising transmission solutions. A multibeam satellite system is suited to provide reliable and low-cost wireless services to rural areas which incumbent terrestrial mobile systems are hard to reach.

In practical satellite operations, one of the issues is the presence of mismatches between the requested traffic and the offered capacity [3]. This is because, firstly, in a multibeam satellite system, the requested traffic from terrestrial terminals are highly asymmetric. Secondly, the satellite resource allocation in multi-beam systems is constrained by satellites' payload design, e.g., limited flexibility in bandwidth, time, or power allocation. As a consequence, it could happen that, in some spot beams, the requested demands from the associated terminals are unmet, resulting in hot beams, whereas the offered capacity in some other beams is unused, leading to cold beams. Both of them are undesirable cases for satellite operators because the former loses revenue corresponding to the unmet demands, and the latter wastes the investment in the unused capacity [3]. Therefore, the capability to overcome this issue by allocating on-board resources over the service coverage is becoming a must for future broadband multi-beam satellites. To well capture the impact of unmet and excess capacity, resource optimization for the Offered Capacity to requested Traffic Ratio (OCTR) has been considered in the literature [3].

As an emerging research area, some efforts have been devoted to introducing $5 \mathrm{G}$ new radio techniques, e.g., Non-Orthogonal Multiple Access (NOMA), to satellite scenarios, in order to further enhance the satellite performance. The major consideration is that, with breaking the orthogonality, multiple terminals in NOMA can access the same time-frequency resource simultaneously, which improves the spectrum efficiency compared to Orthogonal Multiple Access (OMA) in DVB-S2 standards [4]. In addition, performing Multi-User Detection (MUD) and Successive Interference Cancellation (SIC) in NOMA can help to alleviate co-channel interference [5]. Thus NOMA has potentials to enable aggressive frequency reuse but suppress interference at an acceptable level in satellite systems. In [6], NOMA was considered for satellite scenarios for the first time. Two user-scheduling algorithms were proposed in their work to maximize the capacity for over-loaded satellite systems. From a system-level point of view, the authors in [7] analyzed the possibility and applicability of integrating NOMA to satellite systems and provided general approaches for cooperating NOMA with precoding. In [8], the authors considered a satelliteterrestrial system and applied NOMA in the terrestrial part. A joint user pairing, precoding, and power allocation scheme was proposed.

From the literature, NOMA has proven its advantages, e.g., throughput, energy [5], [9], over OMA in terrestrial 
systems. However, for satellite systems, the optimization for the practical metric OCTR is studied to a limited extent, motivating us to fathom this area. The contribution of the paper lies at the following aspects:

- This paper aims at providing answers and algorithmic solutions for the following two research questions: Firstly, is NOMA able to improve the OCTR performance in satellite systems? Secondly, how much is the gain of applying NOMA to the considered problem?

- We consider a max-min resource optimization problem in NOMA-based multi-beam satellite systems, taking OCTR metric and terminals' fairness into account. The problem aims at improving the performance of the terminal with the worst OCTR such that the optimized capacity for terminals can be as close as their requested traffic demands and the fairness among terminals can be improved.

- To solve the problem, firstly we decompose the original problem into multiple convex subproblems by fixing beam power and find the optimum for each beam. Secondly, we iteratively adjust beam power to progressively improve the minimum OCTR.

Numerical results show the convergence of the proposed algorithm. The algorithm also demonstrates the superiority of NOMA over the OMA scheme.

\section{SYSTEM MODEL}

\section{A. Multi-Beam Satellite Systems}

We consider the forward link transmission in a multibeam satellite system, where a Geostationary Earth Orbit (GEO) satellite is equipped with an array-fed reflector antenna with multiple feeds to generate $B$ spot beams (one feed per beam) and provide services to number of $K_{b}$ ground terminals per beam. Let $b=1, \ldots, B$ and $\mathcal{B}$ be the index and set of beams, respectively, where $|\mathcal{B}|=B$. We denote $k=1, \ldots, K_{b}$ and $\mathcal{K}_{b}$ as the index and set of the associated terminals in beam $b$, respectively, where $\left|\mathcal{K}_{b}\right|=K_{b}$. Let $\mathbf{h}_{b k} \in \mathbb{C}^{1 \times B}$ be the channel vector of terminal $k$ in beam $b$. The $m$-th element of $\mathbf{h}_{b k}$, i.e., $h_{b k}^{(m)}$, indicates the channel coefficient from the $m$-th beam feed to terminal $k$ in beam $b$, which can be expressed as $h_{b k}^{(m)}=L_{m b k} G_{b k}^{r} G_{m b k}^{s}$, where $L_{b k}$ is the free-space propagation loss from the $m$-th feed to the $k$ th terminal. $G_{b k}^{r}$ is the $k$-th terminal's receive antenna gain. $G_{m b k}^{s}$ is the gain from the $m$-th feed to the $k$-th terminal in beam $b$. By adopting NOMA, we let all the beams share the same frequency band, i.e., 1-color reuse pattern. In terms of payload, we assume that the on-board payload is equipped with the module of flexible MultiPort Amplifiers (MPAs) such that power can be adjusted.

\section{B. Precoding and NOMA Scheme}

Let $\mathbf{w}_{b} \in \mathbb{C}^{B \times 1}$ denote the precoding vector for beam $b$. For terminal $k$ in beam $b$, the received signal can be expressed as:

$$
\begin{array}{r}
y_{b k}=\underbrace{\mathbf{h}_{b k} \mathbf{w}_{b} \sqrt{p_{b k}} s_{b k}}_{\text {desired signal }}+\underbrace{\mathbf{h}_{b k} \mathbf{w}_{b} \sum_{j \neq k} \sqrt{p_{b j}} s_{b j}}_{\text {intra-beam interference }} \\
+\underbrace{\mathbf{h}_{b k} \sum_{b^{\prime} \neq b} \mathbf{w}_{b^{\prime}} \sum_{l \in \mathcal{K}_{b^{\prime}}} \sqrt{p_{b^{\prime} l}} s_{b^{\prime} l}+n_{b k},}_{\text {inter-beam interference }}
\end{array}
$$

where $p_{b k}, s_{b k}$, and $n_{b k} \sim \mathcal{C N}\left(0, \sigma^{2}\right)$ are the transmit power, the transmit signal with unit power, and the additive noise for terminal $k$ in beam $b$, respectively.

In the paper, we use precoding to reduce inter-beam interference, while NOMA is adopted to eliminate part of intra-beam interference for multiple terminals within a beam. We adopt a linear precoding scheme, Minimum Mean Square Error (MMSE), which is considered with high efficiency and low computational complexity [6], [10]. For MMSE, we denote $\mathbf{H} \in \mathbb{C}^{B \times B}$ as the channel matrix. The $b$-th row represents the channel vector of the terminal with the maximum channel coefficient in beam $b$ [8], i.e., $\arg \max _{k \in \mathcal{K}_{b}}\left\{\left|h_{b k}^{(b)}\right|^{2}\right\}$, where $h_{b k}^{(b)}$ is the channel coefficient of terminal $k$ in beam $b$ when receiving its desired signal from the $b$-th feed. The precoding matrix reads:

$$
\mathbf{W}_{m m s e}=\left(\mathbf{H}^{H} \mathbf{H}+\sigma^{2} \mathbf{I}\right)^{-1} \mathbf{H}^{H},
$$

where $\mathbf{I}$ is the identity matrix. In this paper, we do not discuss precoding design but power allocation for beams and terminals. The power of each precoding vector is then normalized as $\left\|\mathbf{w}_{b}\right\|^{2}=1$.

Within a beam, NOMA is applied to mitigate intrabeam interference among the terminals. According to a widely adopted approach for determining decoding order [11], we define the SIC decoding order as the descending order of the ratio between channel gain to inter-beam interference plus noise, denoted by $g_{b k}$ :

$$
g_{b k}=\frac{\left|\mathbf{h}_{b k} \mathbf{w}_{b}\right|^{2}}{\sum_{b^{\prime} \neq b}\left|\mathbf{h}_{b k} \mathbf{w}_{b^{\prime}}\right|^{2} P_{b^{\prime}}+\sigma^{2}},
$$

where $P_{b^{\prime}}=\sum_{l \in \mathcal{K}_{b^{\prime}}} p_{b^{\prime} l}$. We note that in this paper $g_{b k}$ refers to the channel condition of each terminal. We use vector $\mathbf{P}$ to collect all the beam power $P_{1}, \ldots, P_{b}, \ldots, P_{B}$. The rationale is that, if terminal $j$ can decode the signal of terminal $k$, in order to ensure a successful SIC, the SINR of terminal $k$ 's signal at terminal $j$ 's receiver should be higher than that at terminal $k$, then (3) is used to guarantee this inequality [11].

Based on the NOMA basis [11], a terminal $k$, before decoding its own signal, first performs SIC to decode and subtract the signals from the terminals whose channel conditions are worse than $k$, whereas the signals from the terminals with better channel conditions than $k$ are treated as noise. We define that $\phi_{b}(k)$ is the position of decoding orders in beam $b$. If $\phi_{b}(k)<\phi_{b}(j)$, then $g_{b k}>g_{b j}$ 
and terminal $k$ can decode the signals of terminal $j$. The decoding order position of the terminal with the best channel condition is $\phi_{b}(k)=1$, whose SINR $\gamma_{b k}$ can be expressed as,

$$
\gamma_{b k}=\frac{\left|\mathbf{h}_{b k} \mathbf{w}_{b}\right|^{2} p_{b k}}{\sum_{b^{\prime} \neq b}\left|\mathbf{h}_{b k} \mathbf{w}_{b^{\prime}}\right|^{2} P_{b^{\prime}}+\sigma^{2}} .
$$

This terminal has the highest ratio $g_{b 1}$, and is able to remove all the intra-beam interference. For any terminals with $\phi(k)>1$, the SINR $\gamma_{b k}$ is,

$$
\gamma_{b k}=\frac{\left|\mathbf{h}_{b k} \mathbf{w}_{b}\right|^{2} p_{b k}}{\sum_{\phi\left(k^{\prime}\right)<\phi(k)}\left|\mathbf{h}_{b k} \mathbf{w}_{b}\right|^{2} p_{b k^{\prime}}+\sum_{b^{\prime} \neq b}\left|\mathbf{h}_{b k} \mathbf{w}_{b^{\prime}}\right|^{2} P_{b^{\prime}}+\sigma^{2}} .
$$

The achievable rate of terminal $k$ in beam $b$ is

$$
R_{b k}=\log \left(1+\gamma_{b k}\right)
$$

\section{PROBlEM Formulation}

In this section, we formulate a power allocation problem in $\mathcal{P} 1$ to max-min OCTR among terminals, in which the optimization variables are $p_{b k}, \forall b \in \mathcal{B}, \forall k \in \mathcal{K}_{b}$.

$$
\begin{aligned}
\mathcal{P} 1: & \max _{\left\{p_{b k}\right\}} \min _{b \in \mathcal{B}, k \in \mathcal{K}_{b}}\left\{\frac{R_{b k}}{D_{b k}}\right\} \\
\text { s.t. } & \sum_{b \in \mathcal{B}} \sum_{k \in \mathcal{K}_{b}} p_{b k} \leq P_{t o t}, \\
& \sum_{k \in \mathcal{K}_{b}} p_{b k} \leq P_{b, \max }, \forall b \in \mathcal{B} .
\end{aligned}
$$

In the objective, the OCTR metric for terminal $k$ in beam $b$ is defined as $\frac{R_{b k}}{D_{b k}}$, where $R_{b k}$ and $D_{b k}$ are the offered capacity and the requested traffic, respectively. By optimization, the terminals' fairness can be enhanced by improving the worst-OCTR terminal's performance. Constraint (7b) states that due to the limited on-board power supply, the total power should be less than a budget $P_{\text {tot }}$. In (7c), the allocated power to each feed should be constrained by a peak power $P_{b, \max }$, to avoid nonlinear impairments in on-board high-power amplifiers.

Remark 1. In $\mathcal{P} 1$, the ideal case is $\frac{R_{b k}}{D_{b k}}=1$ for all the terminals. We do not impose constrains as $\frac{R_{b k}}{D_{b k}} \leq 1$ or $\frac{R_{b k}}{D_{b k}} \geq 1$, such that the fluctuation of OCTR around one can be observed.

The problem can be equivalently transformed to $\mathcal{P} 2$ by introducing an auxiliary variable $t$.

$$
\begin{aligned}
\mathcal{P} 2: & \max _{\left\{p_{b k}\right\}, t} t \\
\text { s.t. } & \sum_{b \in \mathcal{B}} \sum_{k \in \mathcal{K}_{b}} p_{b k} \leq P_{\text {tot }}, \\
& \sum_{k \in \mathcal{K}_{b}} p_{b k} \leq P_{b, \max }, \forall b \in \mathcal{B}, \\
& \frac{R_{b k}}{D_{b k}} \geq t, \forall b \in \mathcal{B}, k \in \mathcal{K}_{b} .
\end{aligned}
$$

Some approaches based on standard interference function, e.g., [13], and Perron-Frobenius theory, e.g., [14], were proposed to enable a convergence guaranteed solution to interference control problems. If the decoding order in each beam remains constant for any power allocation, the problem satisfies the specific conditions of these approaches and can be solved. However, in the most general scenarios, it is impractical to keep decoding orders always the same while adjusting transmit power. With the changes of decoding orders, the function $R_{b k}$ is no longer continuous at the point where decoding orders change, which makes the original problem non-convex and much more difficult to solve. Even though the approaches based on standard interference function and Perron-Frobenius theory are mature, these approaches cannot be applied directly since the function $R_{b k}$ is not continuous and does not satisfy the specific conditions of these approaches. Thus we need to decompose the original problem and propose a heuristic algorithm to solve the problem.

\section{Proposed Algorithmic Solution}

To solve $\mathcal{P} 2$, firstly we consider decomposing the optimization task into two levels, i.e., intra-beam and inter-beam. The former is to find the maximum $t_{b}$ within each beam by fixing beam power. The latter aims at adjusting power $P_{1}, \ldots, P_{B}$ among beams to progressively improve the minimum value of $t_{b}$.

\section{A. Power Optimization within Each Beam}

Specifically, we divide $\mathcal{P} 2$ into number of $B$ subproblems with fixed beam power for the moment. In this phase, the decoding order within each beam is fixed. The subproblem for beam $b$ can be formulated as:

$$
\begin{gathered}
\mathcal{P} 3: \max _{p_{b 1}, \ldots, p_{b\left|\mathcal{K}_{b}\right|}, t_{b}} t_{b} \\
\text { s.t. } \sum_{k \in \mathcal{K}_{b}} p_{b k} \leq P_{b}, \\
\\
\quad \frac{R_{b k}}{D_{b k}} \geq t_{b}, \forall k \in \mathcal{K}_{b},
\end{gathered}
$$

where $P_{b}$ in (9b) represents the total allocated power for beam $b$. The value of $P_{b}$ can be further tuned to meet the constraints (7b) and (7c) over iterations. Next, we show that, by fixing the beam power, the allocation problem $\mathcal{P} 3$ is a convex problem. By adopting the substituting method in [5], we can express $p_{b k}$ by a function of $R_{b k}$, e.g., $p_{b 1}=\frac{e^{R_{b 1}}-1}{g_{b 1}}$ and $p_{b 2}=\frac{e^{R_{b 2}}-1}{g_{b 2}}\left(g_{b 2} \frac{e^{R_{b 1}}-1}{g_{b 1}}+1\right)$ for a twoterminal example. $\mathcal{P} 3$ can be equivalently transformed to 
$\mathcal{P} 4$ by treating $R_{b 1}, \ldots, R_{b\left|\mathcal{K}_{b}\right|}$ as variables.

$$
\begin{aligned}
\mathcal{P} 4: \max _{R_{b 1}, \ldots, R_{b\left|\mathcal{K}_{b}\right|}, t_{b}} & t_{b} \\
\text { s.t. } & \sum_{k \in \mathcal{K}_{b}}\left(\frac{1}{g_{b\left[\phi_{b}(k)\right]}}-\frac{1}{g_{b\left[\phi_{b}(k)-1\right]}}\right) e^{\left(\sum_{\phi_{b}(j) \geq \phi_{b}(k)} R_{b j}\right)} \\
& -\frac{1}{g_{b\left[\Phi_{b}\right]}} \leq P_{b},
\end{aligned}
$$

$$
t_{b} D_{b k}-R_{b k} \leq 0, \forall k \in \mathcal{K}_{b},
$$

where we denote $\left[\phi_{b}(k)\right]$ as the index of the terminal whose decoding order position is $\phi_{b}(k) . \Phi_{b}$ denotes the decoding order position of the terminal with the worst channel condition and $\left[\Phi_{b}\right]$ denotes the index of this terminal. Constraint (10b) with the form of sum exponential functions therefore concludes the convexity of (10b) as well as $\mathcal{P} 4$ [12].

\section{B. Power Optimization Among Beams}

Deriving Karush-Kuhn-Tucker (KKT) conditions for $\mathcal{P} 4$, the optimal value of $t_{b}$ can be obtained by the following equation,

$$
\begin{array}{r}
\sum_{k \in \mathcal{K}_{b}}\left(\frac{1}{g_{b\left[\phi_{b}(k)\right]}}-\frac{1}{g_{b\left[\phi_{b}(k)-1\right]}}\right) e^{\left(\sum_{\phi_{b}(j) \geq \phi_{b}(k)} R_{b j}\right)} \\
-\frac{1}{g_{b\left[\Phi_{b}\right]}}-P_{b}=0
\end{array}
$$

where $t_{b} D_{b j}=R_{b j}$ at the optimum. However, the closedform expression of $t_{b}$ by power $P_{1}, \ldots, P_{B}$ is hard to derive. From (11), we then express $t_{b}$ in an implicit way, i.e., $t_{b}(\mathbf{P})=t_{b}\left(P_{1}, \ldots, P_{B}\right)$. Thus, to obtain the maxmin OCTR, the task can be carried out by optimizing $P_{1}, \ldots, P_{B}$ instead of tuning power for each terminal. The corresponding optimization problem $\mathcal{P} 5$ reads,

$$
\begin{aligned}
\mathcal{P} 5 & \max _{P_{1}, \ldots, P_{B}} \min _{b \in \mathcal{B}}\left\{t_{b}\left(P_{1}, \ldots, P_{B}\right)\right\} \\
\text { s.t. } & \sum_{b \in \mathcal{B}} P_{b} \leq P_{\text {tot }}, \\
& P_{b} \leq P_{b, \text { max }}, \forall b \in \mathcal{B} .
\end{aligned}
$$

We remark that $\mathcal{P} 5$ may not be with an appropriate formulation to derive a complete solution due to lack of the explicit expression in the objective. However, deriving a necessary condition for the optimum of $\mathcal{P} 5$ is possible by applying the rule of implicit differentiation. The result is elaborated in Lemma $\mathbf{1}$.

Lemma 1. Suppose $\eta$ is the optimal objective value of $\mathcal{P} 5$, then $t_{b}\left(P_{1}, \ldots, P_{B}\right)=\eta, \forall b \in \mathcal{B}$.

Proof. Suppose $\eta$ is optimal to $\mathcal{P} 5$ and $t_{b}=\eta$ for beam $b$ while $t_{b^{\prime}}>\eta$ for $\forall b^{\prime} \neq b \in \mathcal{B}$. Even though it is hard to obtain the closed-form expression of $t_{b}\left(P_{1}, \ldots, P_{B}\right)$, we can analytically derive the partial derivatives as $\frac{\partial t_{b}(\mathbf{P})}{\partial P_{b}}=$ $\frac{1}{G(\mathbf{P})}$ and $\frac{\partial t_{b}(\mathbf{P})}{\partial P_{b^{\prime}}}=\frac{F(\mathbf{P})}{G(\mathbf{P})}$, where

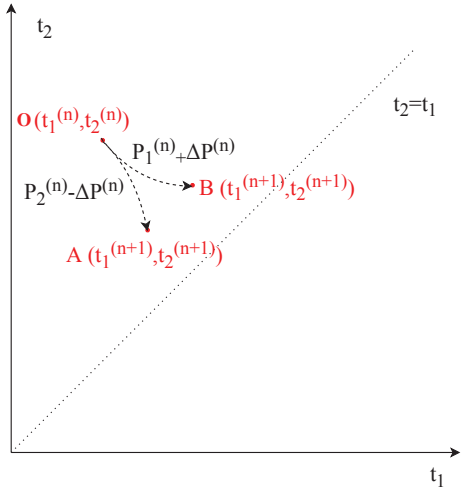

Fig. 1. Illustration of the proposed algorithm.

$$
\begin{array}{r}
G(\mathbf{P})=\sum_{k \in \mathcal{K}_{b}}\left(\frac{1}{g_{b\left[\phi_{b}(k)\right]}}-\frac{1}{g_{b\left[\phi_{b}(k)-1\right]}}\right) \\
\cdot e^{\sum_{\phi(j) \geq \phi(k)} t D_{b j}} \sum_{\phi(j) \geq \phi(k)} D_{b j}, \\
F(\mathbf{P})=\sum_{k \in \mathcal{K}_{b}}\left(\beta_{b\left[\phi_{b}(k)\right] b^{\prime}}-\beta_{b\left[\phi_{b}(k)-1\right] b^{\prime}}\right) \\
\cdot e^{\sum_{\phi(j) \geq \phi(k)} t D_{b j}}-\beta_{b\left[\Phi_{b}\right] b^{\prime}},
\end{array}
$$

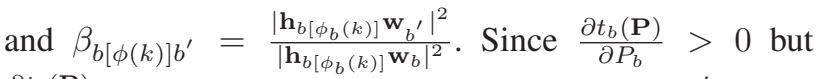
$\frac{\partial t_{b}(\mathbf{P})}{\partial P_{b^{\prime}}}<0$, we can reduce power of any beam $b^{\prime}$ or/and increase power of beam $b$ to raise $t_{b}$. Thus there exists $\eta^{\prime}>\eta$ with a feasible solution, which contradicts the assumption.

\section{The Proposed Algorithm}

The aim of the algorithm is to improve the minimal value of $t_{b}$. Since it is hard to identify the properties of $\mathcal{P} 5$, we propose an iterative methodology to find a suboptimal solution. We will illustrate the methodology with the two-beam case.

From Lemma 1 we know that the necessary condition of obtaining the optimal solution to $\mathcal{P} 5$ is $t_{1}=t_{2}$. Therefore we design the algorithm to increase the value of $\eta=\min _{b=1,2}\left\{t_{b}\right\}$ as much as possible until the condition $t_{1}=t_{2}$ holds. At the $n$-th iteration of the algorithm, if $\eta^{(n)}=t_{1}^{(n)}<t_{2}^{(n)}$, for instance, there are two ways to enhance the value of $\eta: P_{1}^{(n)}+\Delta P^{(n)}$ or $P_{2}^{(n)}-\Delta P^{(n)}$. Suppose the step size $\Delta P^{(n)}$ is small enough. When $P_{2}^{(n)}$ decreases by the value of $\Delta P^{(n)}$, the variations of $t_{1}$ and $t_{2}$ can be approximately derived as: $t_{1}^{(n+1)}-t_{1}^{(n)} \approx-\frac{\partial t_{1}}{\partial P_{2}} \Delta P^{(n)}, t_{2}^{(n+1)}-t_{2}^{(n)} \approx$ $-\frac{\partial t_{2}}{\partial P_{2}} \Delta P^{(n)}$. With the specific precoding method MMSE, $\beta_{b\left[\phi_{b}(k)\right] b^{\prime}}$ is relatively small compared to 1 . So we can derive approximately that $\left|\frac{\partial t_{1}}{\partial P_{2}}\right|<\left|\frac{\partial t_{2}}{\partial P_{2}}\right|$ according to the expressions of the partial derivatives of $t_{b}$. Then $\left|t_{1}^{(n+1)}-t_{1}^{(n)}\right|<\left|t_{2}^{(n+1)}-t_{2}^{(n)}\right|$. Similarly, when $P_{1}^{(n)}$ increases by $\Delta P^{(n)},\left|t_{1}^{(n+1)}-t_{1}^{(n)}\right|>\left|t_{2}^{(n+1)}-t_{2}^{(n)}\right|$. This indicates that enhancing $P_{1}$ can head to the path gaining 


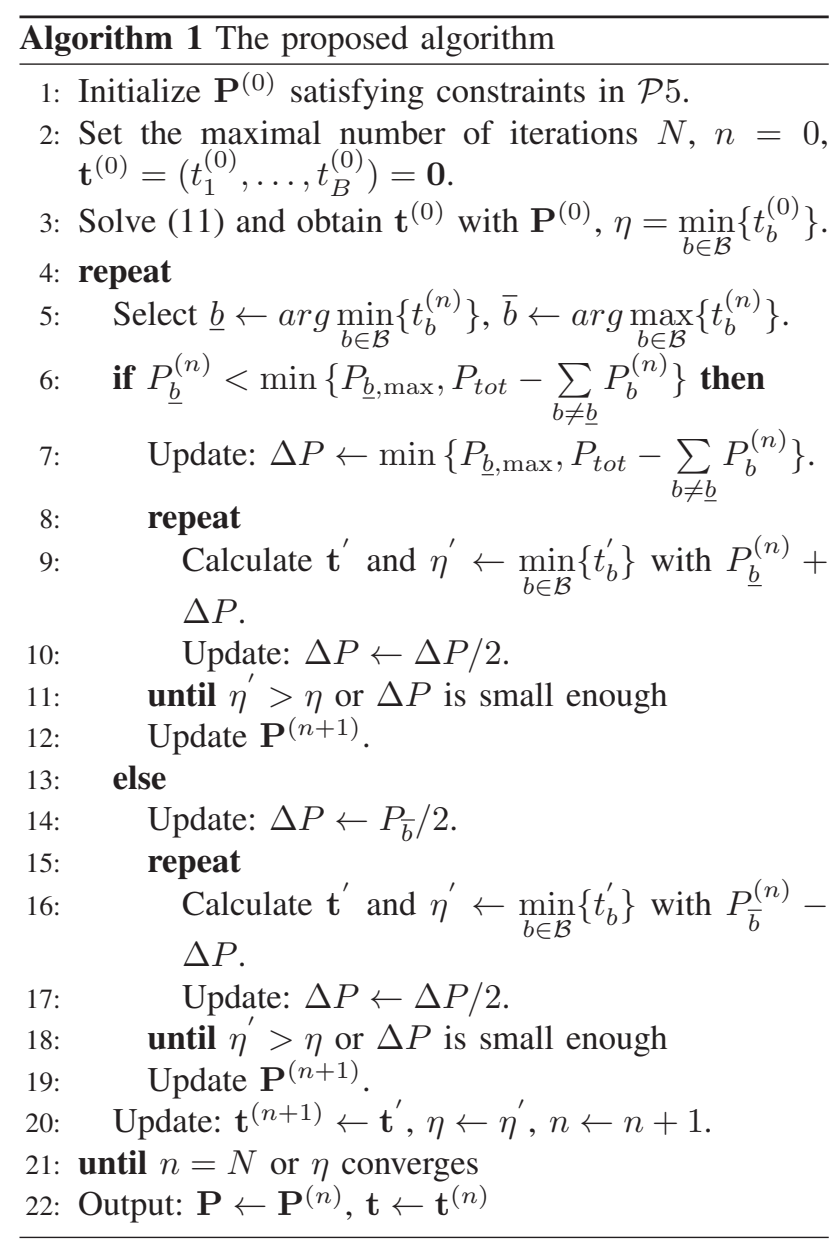

larger $\eta$ than reducing $P_{2}$. The variation tendencies of $t_{1}$ and $t_{2}$ while altering $P_{1}$ and $P_{2}$ are depicted in Fig. 1 . Thus for the beam with smaller $t_{b}$, the effective way to improve $t_{b}$ is to gain more transmit power rather than reduce power of other beams.

The algorithm is described in Alg. 1. At each iteration, it identifies if the power of the beam with the smallest $t_{b}$ is smaller than the maximum limitation. If so, it chooses to improve this beam's power (line 7-12). In this phase, the step size $\Delta P$ is first initialized as the gap between its power level and the maximal power limitation. If $\eta$ gets larger after power rises, $\Delta P$ will be determined; otherwise, $\Delta P$ is halved. If the power of the beam with the smallest $t_{b}$ reaches the maximum limitation, it is designed to reduce power of the beam with the largest $t_{b}$ (line 14-19), in order to enhance the minimal value of $t_{b}$. In this phase, $\Delta P$ can be set as half of its power. $\Delta P$ will be upgraded the same as the process of the first phase.

\section{Performance Evaluation}

In this section, we evaluate the performance of the considered NOMA scheme and the proposed algorithm. The key parameters are summarized in TABLE I. In NOMA, since the MUD receivers' complexity increases

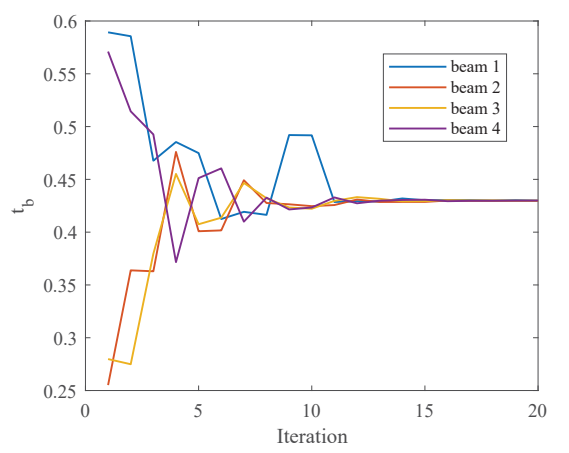

Fig. 2. Evolution of $t_{b}$ in 4 beams over iterations in Algorithm 1

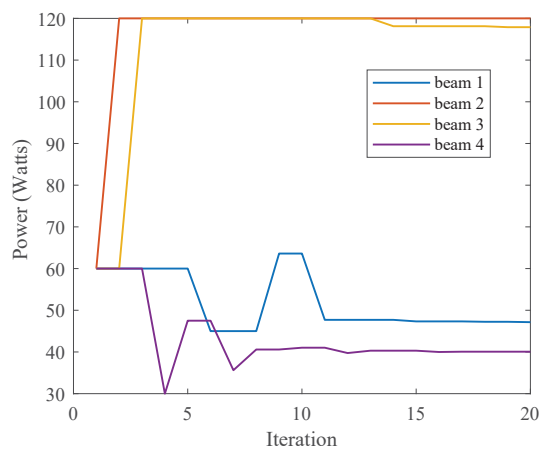

Fig. 3. Evolution of $P_{b}$ in 4 beams over iterations in Algorithm 1

exponentially with the number of signals to be detected [7], in this work we limit the number of terminals per beam to two. In total, we deploy 70 terminals in each beam. At each run of the simulation, we select two of them in each beam. One terminal is chosen randomly first and the other one with large difference of channel gain is then paired [6]. The results are averaged over 1000 instances. The terminals' demands are uniformly distributed between 1 and $5 \mathrm{bits} / \mathrm{s} / \mathrm{Hz}$. To evaluate the performance of the proposed scheme, we adopt OMA with precoding and 1-color reuse pattern, NOMA with 2-color reuse pattern (without precoding), NOMA with 4-color reuse pattern (without precoding), OMA with 1color reuse pattern (without precoding), and NOMA with 1-color reuse pattern (without precoding) for comparison.

Firstly, in Fig. 2 and 3, we evaluate the proposed Algorithm 1 for the NOMA with precoding and 1-color

TABLE I

SimUlation PARAMETERS

\begin{tabular}{|c|c|}
\hline Parameter & Value \\
\hline Frequency & $20 \mathrm{GHz}(\mathrm{Ka}$ band) \\
\hline Bandwidth & $500 \mathrm{MHz}$ \\
\hline Satellite location & $13^{\circ} \mathrm{E}$ \\
\hline Satellite height & $35,786 \mathrm{~km}$ \\
\hline Satellite antenna gain & between 49.60 and $54.63 \mathrm{dBi}$ \\
\hline Receive antenna gain & $42.1 \mathrm{dBi}$ \\
\hline Channel & LoS channel (path loss) \\
\hline Noise power $\left(\sigma^{2}\right)$ & $-126.47 \mathrm{dBW}$ \\
\hline Number of beams $(B)$ & 4 \\
\hline$P_{b, \text { max }}, P_{\text {tot }}$ & $120,400 \mathrm{~W}[14]$ \\
\hline
\end{tabular}




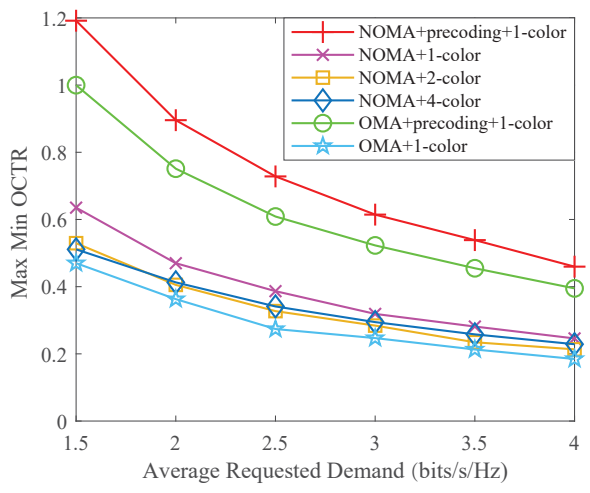

Fig. 4. Max-min OCTR in Algorithm 1 with respect to traffic demand.

reuse pattern. We show the Algorithm 1's evolution over iterations in terms of OCTR value $t_{b}$ and beam power $P_{b}$, respectively. From the two Figures, we can observe that if a beam with lower value of $t_{b}$ in an iteration, e.g., see the curves of beam 2 and 3 in iteration 1 in Fig. 2 and 3, at the next iteration, the algorithm will assign more power to compensate $t_{2}$ and $t_{3}$, whereas the beams 1 and 4 in iteration 1 with the higher OCTR will be assigned by less power in later iterations. From Fig. 2 , the algorithm demonstrates promising performance in convergence. Around 11 iterations, the max-min OCTR value approaches to the convergence point though with the long-tail effect. Analogous to Fig. 2, the power allocation for each beam converges around 13 iterations in Fig. 3.

Next, we use Fig. 4 to show the max-min OCTR performance among six schemes. From the results, the scheme of NOMA with precoding and 1-color reuse pattern outperforms. This is because the scheme is largely benefited from efficient frequency utilization and inter/intrabeam interference mitigation. In addition, when applying NOMA with 1-color reuse pattern, the performance is better than NOMA with 4-color as well as 2-color. The reason can be explained below. In 2-color and 4color schemes, less inter-beam interference presents. To improve the max-min OCTR performance, one way is to increase the transmit power in a beam, say beam $b$. When the transmit power has achieved the peak power limitation $P_{b, \max }$, the other way is then adopted to reduce the inter-beam interference by decreasing power $P_{1}, \ldots, P_{b-1}, P_{b+1}, \ldots, P_{B}$. As a result, when transmit power meets the limitation $P_{b, \max }$, the max-min OCTR performance in 1-color scheme can be further improved by reducing the power in the other beams, whereas the 2/4-color reuse pattern gains less improvement due to less inter-beam interference, thus it results in performance gaps among 1-color, 2-color, and 4-color schemes.

\section{CONCLUSION}

In this paper, we consider a NOMA-based multi-beam satellite system, and address a fairness issue in resource optimization. We aim at maximizing the worst OCTR performance by power optimization, such that the offered and requested traffic can achieve a good match among terminals. The formulated max-min OCTR problem is non-convex in general scenarios. To solve the problem, we decompose the power optimization into each beam, and propose a suboptimal algorithm to enhance the fairness among terminals by sequentially and iteratively optimizing beam power. The simulation results show the convergence of the proposed algorithm, and the performance gain of NOMA than other baseline schemes. The numerical results demonstrate that NOMA is able to enhance the max-min OCTR performance, and the performance could be further improved when more aggressive frequency reuse schemes, e.g., 1-color, are adopted.

\section{ACKNOWLEDGMENT}

The work has been funded by the FNR CORE project ROSETTA (11632107), and by TERESA (TEC201790093-C3-1-R).

\section{REFERENCES}

[1] Cisco, "Cisco visual networking index: Forecast and trends, 20172022 white paper," Feb. 2019.

[2] E. Lagunas, S. Maleki, L. Lei, C. Tsinos, S. Chatzinotas, and B. Ottersten, "Carrier allocation for hybrid satellite-terrestrial backhaul networks," in 2017 IEEE International Conference on Communications Workshops, pp. 718-723.

[3] G. Cocco, T. de Cola, M. Angelone, Z. Katona and S. Erl, "Radio resource management optimization of flexible satellite payloads for DVB-S2 systems," in IEEE Transactions on Broadcasting, vol. 64, no. 2, pp. 266-280, June 2018.

[4] A. Morello and V. Mignone. "DVB-S2: The second generation standard for satellite broad-band services," in Proceedings of the IEEE, 2006, 94(1): 210-227.

[5] L. Lei, D. Yuan, and P. Värbrand, "On power minimization for non-orthogonal multiple access (NOMA)," IEEE Communications Letters, vol. 20, no. 12, pp. 2458-2461, 2016.

[6] M. Caus, M. Á. Vázquez, and A. Pérez-Neira, "NOMA and interference limited satellite scenarios," in 2016 50th Asilomar Conference on Signals, Systems and Computers. IEEE, 2016, pp. $497-501$.

[7] A. I. Pérez-Neira, M. Caus, M. A. Vazquez, and N. Alagha, "NOMA schemes for multibeam satellite communications," arXiv preprint arXiv:1810.08440, 2018.

[8] X. Zhu, C. Jiang, L. Kuang, N. Ge, and J. Lu, "Non-orthogonal multiple access based integrated terrestrial-satellite networks,' IEEE Journal on Selected Areas in Communications, vol. 35, no. 10 , pp. 2253-2267, 2017.

[9] L. Lei et al., "Learning-assisted optimization for energy-efficient scheduling in deadline-aware NOMA systems," in IEEE Transactions on Green Communications and Networking.

[10] T. K. Lyu, "Capacity of multi-user MIMO systems with MMSE and ZF precoding," in 2016 IEEE Conference on Computer Communications Workshops. IEEE, 2016, pp. 1083-1084.

[11] S. R. Islam, N. Avazov, O. A. Dobre, and K.-S. Kwak, "Powerdomain non-orthogonal multiple access (NOMA) in 5G systems: Potentials and challenges," IEEE Communications Surveys \& Tutorials, vol. 19, no. 2, pp. 721-742, 2017.

[12] S. Boyd and L. Vandenberghe, Convex Optimization, Cambridge, UK: Cambridge University Press, 2004.

[13] Yates, Roy D. "A framework for uplink power control in cellular radio systems." IEEE Journal on Selected Areas in Communications, 13.7 (1995): 1341-1347.

[14] A. I. Aravanis, B. Shankar M. R., P. Arapoglou, G. Danoy, P. G. Cottis and B. Ottersten, "Power allocation in multibeam satellite systems: a two-stage multi-objective optimization," in IEEE Transactions on Wireless Communications, vol. 14, no. 6 , pp. 3171-3182, June 2015. 